\title{
Technical note: Validation of the BHBCheck blood $\beta$-hydroxybutyrate meter as a diagnostic tool for hyperketonemia in dairy cows
}

\author{
K. J. Sailer, ${ }^{*}$ R. S. Pralle, ${ }^{*}$ R. C. Oliveira, ${ }^{*}$ S. J. Erb, ${ }^{*}$ G. R. Oetzel,† and H. M. White ${ }^{* 1}$ \\ *Department of Dairy Science, and \\ †School of Veterinary Medicine, University of Wisconsin-Madison, Madison 53706
}

\begin{abstract}
Accurate cow-side blood $\beta$-hydroxybutyrate (BHB) detection meters are valuable tools for rapid diagnosis of hyperketonemia. The main objective of this study was to compare the blood BHB measured in whole blood by the BHBCheck meter (PortaCheck, Moorestown, NJ) to a previously validated meter, Precision Xtra meter (Abbott Laboratories, Abbott Park, IL) and a colorimetric laboratory assay. Samples $(\mathrm{n}=426)$ were collected from postpartum primiparous and multiparous Holstein cows ( $\mathrm{n}=79$ cows) enrolled in 1 of 2 experiments (Exp) with different sampling schedules (Exp 1: $\mathrm{n}=39$ cows, 58 samples; Exp 2: $\mathrm{n}=40$ cows, 368 samples). In both Exp, whole-blood samples were collected from the coccygeal vessels after morning milking, before morning feeding. Blood samples were used immediately for BHB quantification via the BHBCheck meter and the Precision Xtra meter. Blood was also collected into evacuated tubes containing no additive (Exp 1) or potassium oxalate/sodium fluoride (Exp 2), which were centrifuged for serum or plasma separation and stored at $-20^{\circ} \mathrm{C}$ for subsequent analysis. Laboratory quantification of $\mathrm{BHB}$ concentration was done by the BHB LiquiColor Assay (EKF Diagnostics-Stanbio, Boerne, TX; certified for serum and plasma). Data were analyzed by UNIVARIATE, CORR, FREQ, REG, and LOGISTIC procedures of SAS 9.4 (SAS Institute Inc., Cary, NC). Within this sample set, average parity was 3.3 lactations and DIM was $14 \mathrm{~d}$. The proportion of samples classified as hyperketonemia (BHB $\geq 1.2$ $\mathrm{mmol} / \mathrm{L}$ ) was 25,28 , and $31 \%$ as determined by the colorimetric assay, BHBCheck meter, and Precision Xtra meter, respectively. The correlation for BHBCheck meter BHB concentration compared with the colorimetric assay concentrations was $\mathrm{r}=0.96$, with a sensitivity of $91 \%$ and specificity of $93 \%$. Correlation, sensitivity, and specificity of the Precision Xtra meter concentrations
\end{abstract}

Received July 27, 2017.

Accepted October 26, 2017.

${ }^{1}$ Corresponding author: heather.white@wisc.edu were $0.97,98 \%$, and $92 \%$, respectively. Bland-Altman plots demonstrated minimal bias for both meters. Area under the receiver operator characteristic curve suggests adequate diagnostic accuracy of both meters. Overall, accuracy, sensitivity, and specificity of the BHBCheck meter was similar to the Precision Xtra meter and laboratory assay, indicating the BHBCheck meter is appropriate for use as a cow-side diagnostic test for hyperketonemia in dairy cows.

Key words: ketosis, cow-side diagnostic tool, transition cow

\section{Technical Note}

During the postpartum period, dairy cows are at increased risk of developing metabolic disorders, largely due to negative energy balance and subsequent mobilization of triglyceride (TG) from adipose tissue (Grummer, 1993; Drackley, 1999; Duffield, 2000). Fates of mobilized TG within the liver include complete oxidation to energy, incomplete oxidation to ketone bodies, or storage as TG (Grummer, 1993). Although some peripheral tissues can use ketone bodies as an energy source, production of ketone bodies beyond tissue usage results in hyperketonemia (HYK). When untreated, HYK is associated with negative effects on animal health, production, and profitability (Herdt, 2000; McArt et al., 2015). Hyperketonemia is defined as blood BHB $\geq 1.2 \mathrm{mmol} / \mathrm{L}$ (Iwersen et al., 2009; McArt et al., 2012b; Gordon et al., 2013, 2017), and the average postpartum prevalence of HYK worldwide ranges from 15 to $22 \%$, although it is highly variable by farm (Suthar et al., 2013; Chandler et al., 2015; Santschi et al., 2016). The average cost per case is $\$ 289$ (McArt et al., 2015); however, early treatment can reduce the costs and negative outcomes (McArt et al., 2012a), making detection protocols that are composed of labor- and cost-effective diagnostics essential to managing HYK.

Previous validation of a handheld blood ketone meter, the Precision Xtra meter (Abbott Laboratories, Abbott Park, IL), provided a valuable quantitative tool for diagnosing HYK using a minimally invasive blood sample 
with greater sensitivity and specificity than previously used milk and urine tests (Oetzel, 2004; Iwersen et al., 2009). Recently, several additional meters have been developed and some have been evaluated for potential use in bovine HYK diagnostics (Bach et al., 2016). Validation of these diagnostic tests in a controlled setting, before field application, is essential. A relatively new meter that has not yet been validated for use in bovine HYK diagnostics is the BHBCheck meter (PortaCheck, Moorestown, NJ). This meter quantifies BHB via a 0.7$\mu \mathrm{L}$ whole-blood sample, which is less blood than other meters (which require between 0.8 to $1.5 \mu \mathrm{L}$ of blood), and quantification is completed in under $5 \mathrm{~s}$ compared with other meters that average $10 \mathrm{~s}$. We hypothesized that, similar to other handheld BHB diagnostic meters, the BHBCheck meter would provide adequate accuracy for HYK diagnostics in dairy cattle. The objectives of our study were (1) to determine the efficacy of the BHBCheck meter as a cow-side BHB-monitoring device by validating it against a colorimetric laboratory assay and (2) to compare the BHBCheck meter to an industry standard cow-side meter (Precision Xtra).

We conducted 2 animal experiments that were approved by the University of Wisconsin-Madison College of Agricultural and Life Sciences Animal Care and Use Committee (protocol numbers A05467 and A01569). For both experiments (Exp), blood was collected from Holstein cows immediately following morning milking and before feeding. The samples from Exp 1 were collected from 39 primiparous and multiparous cows between 4 and 18 DIM during twice-weekly sampling on a privately owned dairy in south-central Wisconsin from May to June 2016. Experiment 1 was intended to provide a balanced data set of an equal number of samples that were above and below the $1.2 \mathrm{mmol} / \mathrm{L} \mathrm{HYK}$ threshold. This was accomplished by collecting whole blood from cows between 4 and 18 DIM into evacuated collection tubes without additives and immediately analyzing blood using the Precision Xtra test. When a cow with HYK was identified, she was included in the study along with the next cow that was negative for HYK. Whole-blood samples were also analyzed using the BHBCheck meter. Samples were collected over several farm visits and some cows contributed more than 1 sample to the data set. Although the balanced design in Exp 1 resulted in a nonrepresentative sample set relative to the population prevalence, it avoided having a sample set with a minimal proportion of samples above the $1.2 \mathrm{mmol} / \mathrm{L}$ threshold. Whole-blood tubes were then centrifuged $\left(2,500 \times g, 15^{\circ} \mathrm{C}, 15 \mathrm{~min}\right)$ and serum was aliquoted and stored in $1.5-\mathrm{mL}$ microtubes at $-20^{\circ} \mathrm{C}$ until subsequent analysis. Experiment 2 was conducted at the University of Wisconsin-Madison Dairy Cattle Center from May to October 2016. Blood samples were collected from 40 multiparous cows between 1 and 45 DIM into an evacuated tube without additives as well as into an evacuated tube containing potassium oxalate and sodium fluoride. Whole blood from the tube without additives was immediately analyzed on the BHBCheck and Precision Xtra meter. The tube containing potassium oxalate and sodium fluoride was centrifuged $\left(2,000 \times g, 4^{\circ} \mathrm{C}, 15 \mathrm{~min}\right)$ and plasma was aliquoted into $1.5-\mathrm{mL}$ microtubes and stored at $-20^{\circ} \mathrm{C}$ for subsequent analysis.

Serum samples from Exp 1 and plasma samples from Exp 2 were analyzed using the LiquiColor colorimetric assay (EKF Diagnostics-Stanbio, Boerne, TX; certified for serum and plasma collected with EDTA, heparin, or sodium fluoride) per the manufacturer's protocol. Samples were quantified in duplicate and samples with intra-assay coefficient of variation greater than $10 \%$ were reanalyzed. The interassay coefficient of variation of the laboratory assay was $6.7 \%$. Sample BHB concentration determined by the laboratory assay served as the gold standard BHB concentration for statistical analysis.

Data analysis was completed in SAS 9.4 (SAS Institute Inc., Cary, NC). Descriptive statistics were analyzed by PROC UNIVARIATE. Residuals were produced by PROC REG with colorimetric laboratory assay BHB as the dependent variable and BHBCheck or Precision Xtra meter-quantified BHB as the independent variable. Pearson correlations between the BHB concentrations determined by both meters and by the colorimetric assay were analyzed by PROC CORR. To account for correlation among residual errors due to the repeated measures on the experimental unit (cow) across time, different error structures (i.e., compound symmetry, first-order autoregressive, and spatial power) were tested using PROC MIXED. A log-likelihood ratio test was performed between the repeated measure models and the null model and indicated accounting for repeated structure of the data did not significantly alter model fit; therefore, analysis of the data continued without accounting for repeated measures. Sensitivity and specificity were calculated by PROC FREQ. Receiver operating characteristic (ROC) curves and associated area under the curve were determined by PROC LOGISTIC. Results for Exp 1 and 2 are provided both separately and combined to provide the diagnostic accuracy of the meters in 2 sample sets with different proportion of samples $\geq 1.2 \mathrm{mmol} / \mathrm{L}$.

Demographics of the samples from Exp 1, 2, and overall are provided in Table 1. Samples were collected from 79 Holstein cows with an average parity of $3.3 \pm$ 0.1 and average DIM of $14.3 \pm 0.6$ across the 2 experiments (Table 1). The mean BHB was $1.3 \pm 0.12,1.0 \pm$ 0.03 , and $1.0 \pm 0.03 \mathrm{mmol} / \mathrm{L}$ for samples from Exp 1, 
Table 1. Demographics of samples from experiment (Exp) 1 (collected between 4 and 18 DIM), Exp 2 (collected between 1 and 45 DIM), and overall used to evaluate the cow-side blood BHB meters ${ }^{1}$ compared with a colorimetric laboratory assay ${ }^{2}$

\begin{tabular}{lccc}
\hline Item & Exp 1 & Exp 2 & Overall \\
\hline Unique cows, no. & \multicolumn{1}{c}{39} & 40 & 79 \\
Samples, no. & 58 & 368 & 426 \\
Cow variable & & & \\
Parity \pm SE & $2.7 \pm 0.18$ & $3.3 \pm 0.08$ & $3.3 \pm 0.08$ \\
DIM \pm SE & $10.2 \pm 0.56$ & $15.1 \pm 0.66$ & $14.3 \pm 0.58$ \\
BHB ${ }^{3}$ mmol/L & & & \\
Mean \pm SE & $1.3 \pm 0.12$ & $1.0 \pm 0.03$ & $1.0 \pm 0.03$ \\
Median & 1.2 & 0.7 & 0.8 \\
Minimum & 0.3 & 0.3 & 0.3 \\
Maximum & 3.9 & 5.4 & 5.4 \\
\hline
\end{tabular}

${ }^{1}$ BHBCheck meter (PortaCheck, Moorestown, NJ) and Precision Xtra meter (Abbott Laboratories, Abbott Park, IL).

${ }^{2}$ LiquiColor colorimetric assay (EKF Diagnostics-Stanbio, Boerne, TX).

${ }^{3}$ As quantified by the colorimetric laboratory assay.

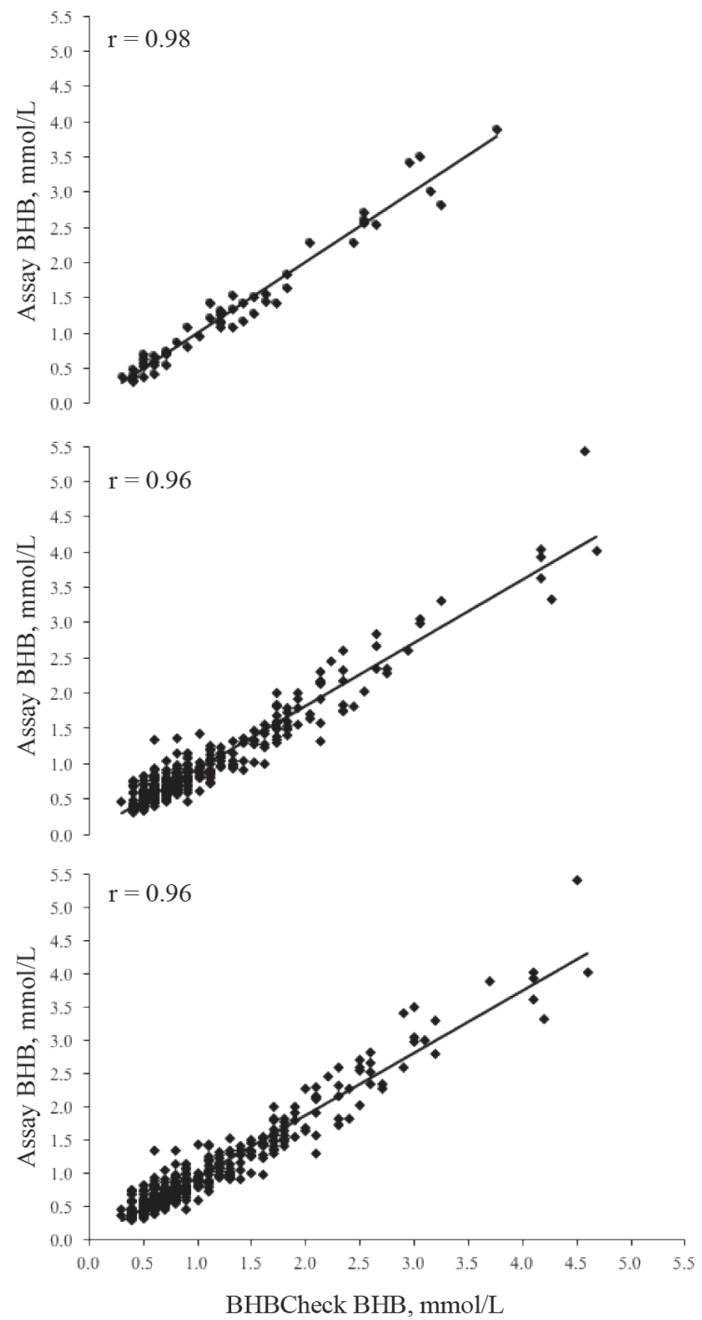

2 , and overall, respectively. The range of BHB was from 0.3 to $5.4 \mathrm{mmol} / \mathrm{L}$ across all samples, as determined by colorimetric laboratory assay.

Proportion of samples with $\mathrm{BHB} \geq 1.2 \mathrm{mmol} / \mathrm{L}$, as determined by the colorimetric assay, were $50 \%$ for Exp 1, 21\% for Exp 2, and $25 \%$ for the overall data set. Use of either meter to calculate the proportion of samples with $\mathrm{BHB} \geq 1.2 \mathrm{mmol} / \mathrm{L}$ resulted in slight overestimations in Exp 2 and overall for the BHBCheck (50, 25, and $28 \%$ for Exp 1, 2, and overall) and in Exp 1, 2, and overall for the Precision Xtra $(55,27$, and $31 \%$ for Exp 1,2 , and overall). This overestimation highlights the possibility of false-positive results when using a cowside BHB meter to diagnose HYK and would result in treating a small number of cows that do not have blood $\mathrm{BHB} \geq 1.2 \mathrm{mmol} / \mathrm{L}$ per the laboratory assay. Treat-
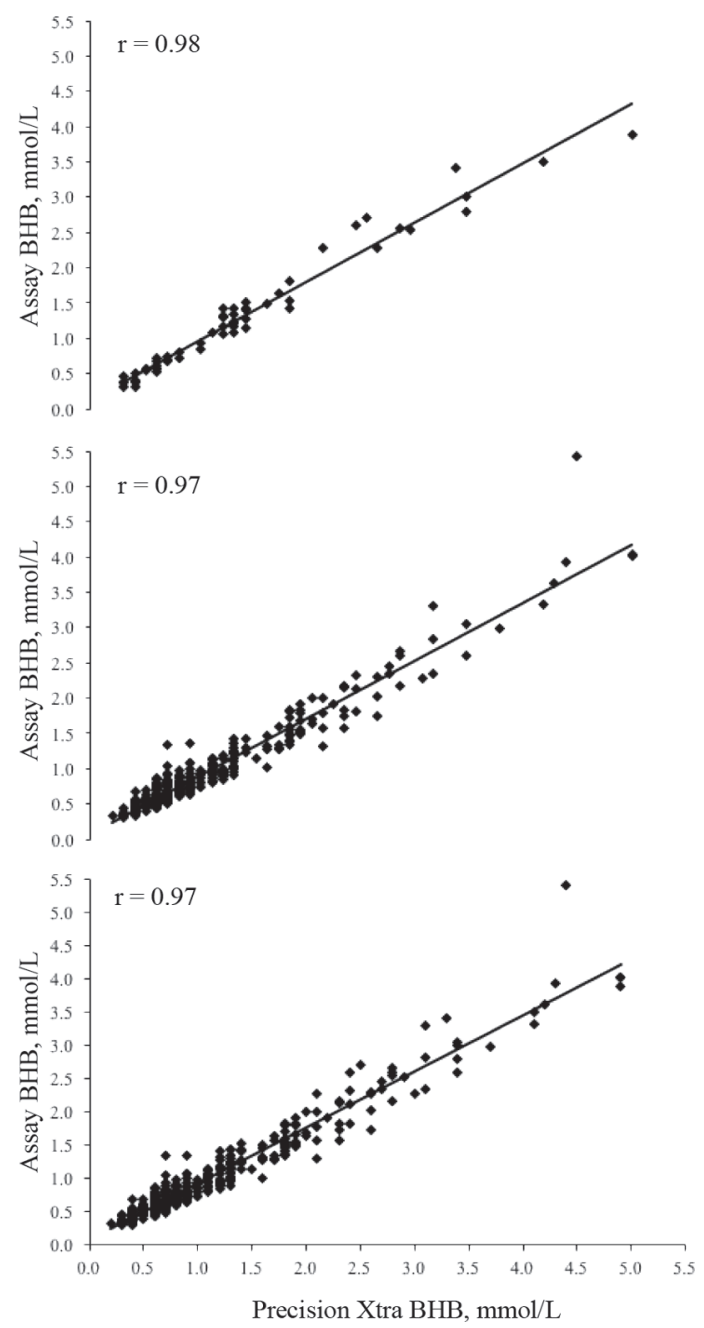

Figure 1. Linear regression of blood BHB concentrations quantified by BHBCheck (PortaCheck, Moorestown, NJ; left panels) and Precision Xtra meter (Abbott Laboratories, Abbott Park, IL; right panels) compared with a colorimetric laboratory assay (LiquiColor colorimetric assay; EKF Diagnostics-Stanbio, Boerne, TX) within experiment (Exp) 1 (58 samples, collected between 4 and 18 DIM; top panels), Exp 2 (368 samples, collected between 1 and 45 DIM; middle panels), and overall (bottom panels). 

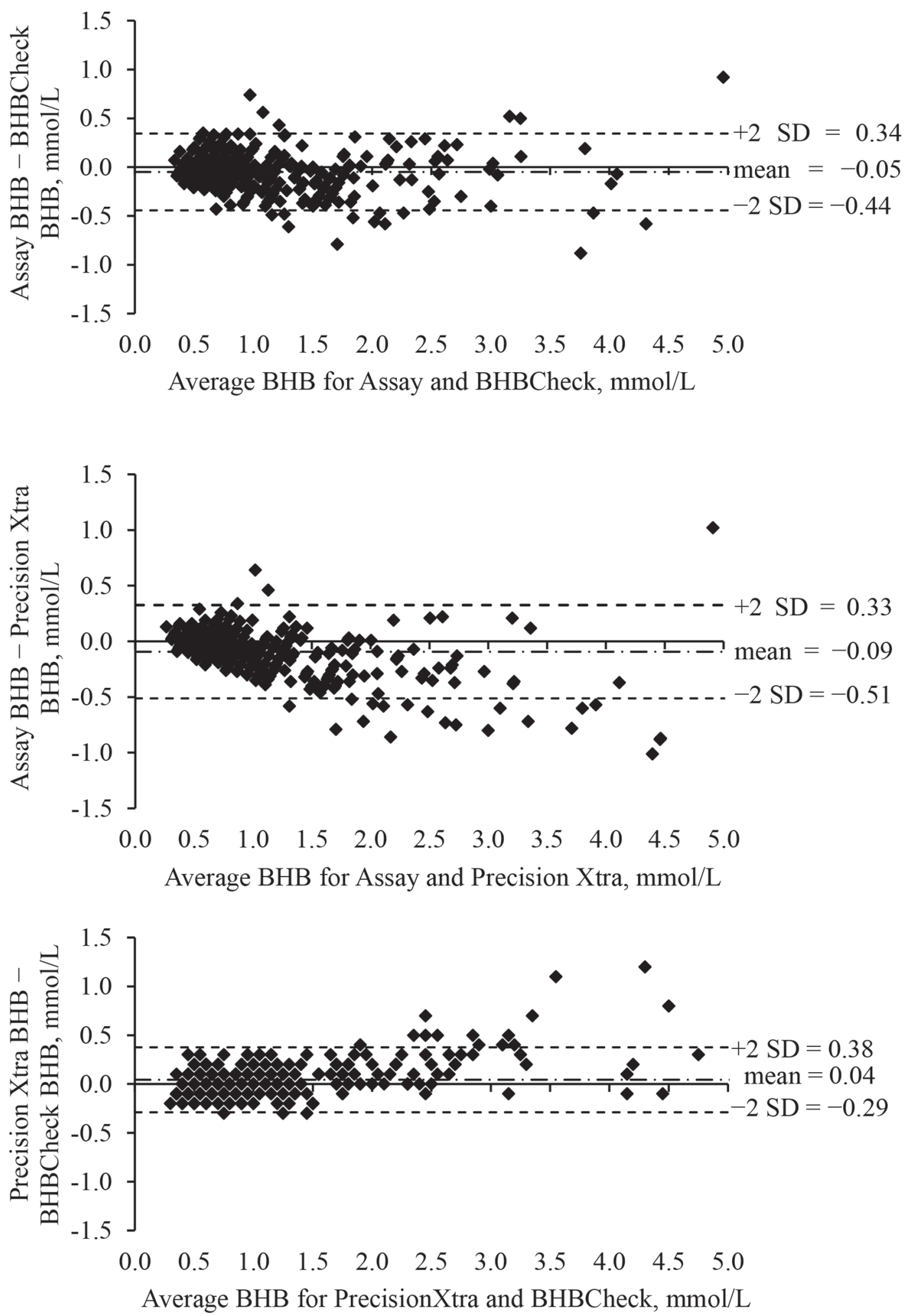

Figure 2. Differences of blood BHB concentrations determined in 426 samples (collected between 1 and 45 DIM across 2 studies) by a laboratory assay (LiquiColor colorimetric assay; EKF Diagnostics-Stanbio, Boerne, TX) and BHBCheck (PortaCheck, Moorestown, NJ; top panel) or Precision Xtra meter (Abbott Laboratories, Abbott Park, IL; middle panel) and between the 2 meters (bottom panel). 
ing false-positives does have a cost associated with it depending on the disease and treatment; however, in the case of HYK, the cost of treatment is relatively low compared with that of an untreated HYK case (McArt et al., 2015). Concentrations of BHB as determined by the BHBCheck or Precision Xtra meter were correlated $(P<0.001)$ with concentrations determined by the colorimetric laboratory assay. Correlation coefficients were 0.98 for both meters compared with the laboratory assay in Exp 1. The correlation coefficients were 0.96 and 0.97 for the BHBCheck and Precision Xtra meter, respectively, in Exp 2 and the overall data set. Interestingly, the correlation between the 2 meters was 0.98 for $\operatorname{Exp} 1,2$, and overall. Fit statistics for linear regression analysis of the BHBCheck and laboratory assay resulted in a correlation coefficient and root mean square error of 0.96 and 0.17 for Exp 1, 0.92 and 0.19 for Exp 2, and 0.93 and 0.19 overall, respectively (Figure 1).

Linear regression analysis of the Precision Xtra meter compared with the laboratory assay resulted in a correlation coefficient and root mean square error values of 0.97 and 0.17 for Exp 1, 0.94 and 0.17 for Exp 2, and 0.94 and 0.17 overall, respectively (Figure 1). BlandAltman plots (Bland and Altman, 1986) showed test agreement (Figure 2) and demonstrated minimal bias for both meters $(-0.05$ and -0.09 for the BHBCheck and Precision Xtra meter, respectively, compared with the laboratory assay). Bias of -0.05 and -0.09 did not influence diagnostic ability of the test cow-side and is consistent with minimal bias previously observed with cow-side BHB diagnostic tests (Bach et al., 2016).

Although correlation and regression methods are valuable indicators of a diagnostic tool's quantitative accuracy, it is equally important to evaluate the sensitivity and specificity of the meters at relevant diagnostic cutoff concentrations. Sensitivity and specificity of the Precision Xtra meter were 98 and $92 \%$ at 1.2 $\mathrm{mmol} / \mathrm{L}$, respectively, for the overall data set in the current study (Table 2), which is consistent with previous validation of this meter (Iwersen et al., 2009; Voyvoda and Erdogan, 2010; Bach et al., 2016). Similarly, the BHBCheck meter had sensitivity and specificity of 91 and $93 \%$ at the $1.2 \mathrm{mmol} / \mathrm{L}$ threshold, respectively. Sensitivity and specificity were greater in Exp 2 than Exp 1, likely due to the difference in sample size. Although both sensitivity and specificity are important indicators of a diagnostic tool, sensitivity is arguably more valuable for subclinical disorders with low-cost and low-risk treatments. Subclinical animals would fail to be diagnosed if missed by the diagnostic test, given the lack of obvious disease symptoms. In the case of HYK, the cost of treatment is relatively low compared with the cost of an untreated case (McArt et al., 2015); thus, adequate sensitivity of a potential cow-side diagnostic tool is important to consider (Bach et al., 2016).

Determination of the area under the ROC curve is an objective means of evaluating the overall validity of a diagnostic tool (Šimundić, 2009). Receiver operating characteristic curves represent the paired sensitivity and specificity for each cutoff; when plotted, the resulting curve allows for estimation of the discriminative power of a test. Areas under the ROC curve between 0.9 and 1.0 represent excellent diagnostic accuracy according to ROC diagnostic categories (Šimundić, 2009). In the current study, area under the ROC curve for the BHBCheck meter was 0.98, 0.98, and 0.98 for Exp 1, 2, and overall, respectively; and area under the ROC curve for the Precision Xtra meter was 0.98, 0.99, and 0.99 for Exp 1, 2, and overall, respectively. This evaluation indicates that both meters portray adequate diagnostic abilities.

Overall, performance of the Precision Xtra meter within the current study was consistent with that in previous studies (Iwersen et al., 2009; Bach et al., 2016). The Precision Xtra meter has been previously validated and has set the expectation for sensitivity and specificity for a cow-side BHB meter. Although it is advantageous to have several diagnostic options, diagnostic tools need to be evaluated to ensure test accuracy. Within the current study, the BHBCheck meter performed similarly to the Precision Xtra meter in our study and to other meters previously examined (Iwersen et al., 2009; Voyvoda and Erdogan, 2010; Bach et al., 2016). Both meters were consistent with expectations of a diagnostic tool and displayed adequate diagnostic accuracy. We conclude that the BHBCheck meter is an accurate and valuable option for use in HYK detection protocols.

Table 2. Sensitivity and specificity of cow-side blood BHB meters ${ }^{1}$ to correctly identify samples as $>1.2 \mathrm{mmol} / \mathrm{L}$, based on a colorimetric laboratory assay ${ }^{2}$ within experiment (Exp) 1 (collected between 4 and 18 DIM), Exp 2 (collected between 1 and 45 DIM), and overall $(95 \%$ CI in parentheses)

\begin{tabular}{llll}
\hline Item & \multicolumn{1}{c}{$\operatorname{Exp~1}$} & Exp 2 & Overall \\
\hline Samples, no. & 58 & 368 & 426 \\
Samples $\geq 1.2$ mmol/L, no. & 29 & 77 & 106 \\
BHBCheck & & & \\
$\quad$ Sensitivity, \% & $89(71-98)$ & $91(84-96)$ & $91(84-96)$ \\
Specificity, \% & $84(66-95)$ & $93(89-95)$ & $93(89-95)$ \\
Precision Xtra & & & \\
Sensitivity, \% & $100(87-100)$ & $97(91-99)$ & $98(93-99)$ \\
Specificity, \% & $84(66-95)$ & $92(88-95)$ & $92(88-94)$ \\
\hline
\end{tabular}

${ }^{1}$ BHBCheck meter (PortaCheck, Moorestown, NJ) and Precision Xtra meter (Abbott Laboratories, Abbott Park, IL).

${ }^{2}$ LiquiColor colorimetric assay (EKF Diagnostics-Stanbio, Boerne, TX). 


\section{ACKNOWLEDGMENTS}

This research was partially funded by gifts from the Wisconsin Alumni Research Foundation (Madison). BHBCheck meters and strips were provided by PortaCheck (Moorestown, NJ). The authors thank the University of Wisconsin-Madison Dairy Cattle Center staff as well as the owner and herdsman of the privately owned dairy for their cooperation during this research project.

\section{REFERENCES}

Bach, K. D., W. Heuwieser, and J. A. A. McArt. 2016. Technical note: Comparison of 4 electronic handheld meters for diagnosing hyperketonemia in dairy cows. J. Dairy Sci. 99:9136-9142. https://doi .org/10.3168/jds.2016-11077.

Bland, J. M., and D. G. Altman. 1986. Statistical methods for assessing agreement between two methods of clinical measurement. Lancet 1:307-310.

Chandler, T. L., R. S. Pralle, G. R. Oetzel, R. H. Fourdraine, and H. M. White. 2015. Development of a ketosis prevalence tool in Holstein dairy cows based on milk component data and cow test-day information. J. Dairy Sci. 98(Suppl 2.):507. (Abstr.)

Drackley, J. K. 1999. ADSA Foundation Scholar Award. Biology of dairy cows during the transition period: The final frontier? J. Dairy Sci. 82:2259-2273. https://doi.org/10.3168/jds.S0022 $-0302(99) 75474-3$.

Duffield, T. 2000. Subclinical ketosis in lactating dairy cattle. Vet. Clin. North Am. Food Anim. Pract. 16:231-253.

Gordon, J. L., T. F. Duffield, T. H. Herdt, D. F. Kelton, L. Neuder, and S. J. LeBlanc. 2017. Effects of a combination butaphosphan and cyanocobalamin product and insulin on ketosis resolution and milk production. J. Dairy Sci. 100:2954-2966. https://doi.org/10 .3168/jds.2016-11925.

Gordon, J. L., S. J. Leblanc, and T. F. Duffield. 2013. Ketosis treatment in lactating dairy cattle. Vet. Clin. North Am. Food Anim. Pract. 29:433-445. https://doi.org/10.1016/j.cvfa.2013.03.001.
Grummer, R. R. 1993. Etiology of lipid-related metabolic disorders in periparturient dairy cows. J. Dairy Sci. 76:3882-3896.

Herdt, T. H. 2000. Ruminant adaptation to negative energy balance. Influences on the etiology of ketosis and fatty liver. Vet. Clin. North Am. Food Anim. Pract. 16:215-230. https://doi.org/10 .1016/S0749-0720(15)30102-X.

Iwersen, M., U. Falkenberg, R. Voigtsberger, D. Forderung, and W. Heuwieser. 2009. Evaluation of an electronic cowside test to detect subclinical ketosis in dairy cows. J. Dairy Sci. 92:2618-2624. https://doi.org/10.3168/jds.2008-1795.

McArt, J. A. A., D. V. Nydam, and G. R. Oetzel. 2012a. A field trial on the effect of propylene glycol on displaced abomasum, removal from herd, and reproduction in fresh cows diagnosed with subclinical ketosis. J. Dairy Sci. 95:2505-2512. https://doi.org/10.3168/ jds.2011-4908.

McArt, J. A. A., D. V. Nydam, and G. R. Oetzel. 2012b. Epidemiology of subclinical ketosis in early lactation dairy cattle. J. Dairy Sci. 95:5056-5066. https://doi.org/10.3168/jds.2012-5443.

McArt, J. A. A., D. V. Nydam, and M. W. Overton. 2015. Hyperketonemia in early lactation dairy cattle: A deterministic estimate of component and total cost per case. J. Dairy Sci. 98:2043-2054. https://doi.org/10.3168/jds.2014-8740.

Oetzel, G. R. 2004. Monitoring and testing dairy herds for metabolic disease. Vet. Clin. North Am. Food Anim. Pract. 20:651-674. https://doi.org/10.1016/j.cvfa.2004.06.006.

Santschi, D. E., R. Lacroix, J. Durocher, M. Duplessis, R. K Moore, and D. M. Lefebvre. 2016. Prevalence of elevated milk $\beta$-hydroxybutyrate concentrations in Holstein cows measured by Fourier-transform infrared analysis in Dairy Herd Improvement milk samples and association with milk yield and components. J. Dairy Sci. 99:9263-9270. https://doi.org/10.3168/jds.2016-11128.

Šimundić, A. M. 2009. Measures of diagnostic accuracy: Basic definitions. EJIFCC 19:203-211.

Suthar, V. S., J. Canelas-Raposo, A. Deniz, and W. Heuwieser. 2013. Prevalence of subclinical ketosis and relationships with postpartum diseases in European dairy cows. J. Dairy Sci. 96:2925-2938. https://doi.org/10.3168/jds.2012-6035.

Voyvoda, H., and H. Erdogan. 2010. Use of a hand-held meter for detecting subclinical ketosis in dairy cows. Res. Vet. Sci. 89:344-351. https://doi.org/10.1016/j.rvsc.2010.04.007. 